Marquette University

e-Publications@Marquette

Biomedical Engineering Faculty Research and

Publications

Biomedical Engineering, Department of

$12-2004$

\title{
Quantitative Models of the Rat Pulmonary Arterial Tree Morphometry Applied to Hypoxia-Induced Arterial Remodeling
}

Robert C. Molthen

Marquette University, robert.molthen@marquette.edu

Kelly L. Karau

Marquette University

Christopher A. Dawson

Medical College of Wisconsin

Follow this and additional works at: https://epublications.marquette.edu/bioengin_fac

Part of the Biomedical Engineering and Bioengineering Commons

\section{Recommended Citation}

Molthen, Robert C.; Karau, Kelly L.; and Dawson, Christopher A., "Quantitative Models of the Rat Pulmonary Arterial Tree Morphometry Applied to Hypoxia-Induced Arterial Remodeling" (2004). Biomedical Engineering Faculty Research and Publications. 113.

https://epublications.marquette.edu/bioengin_fac/113 
Marquette University

e-Publications@Marquette

\section{Biomedical Engineering Faculty Research and Publications/College of} Engineering

This paper is NOT THE PUBLISHED VERSION; but the author's final, peer-reviewed manuscript. The published version may be accessed by following the link in the citation below.

Journal of Applied Physiology, Vol. 97, No. 6 (December 2004): 2372-2384. DOI. This article is (C) American Physiological Society and permission has been granted for this version to appear in ePublications@Marquette. American Physiological Society does not grant permission for this article to be further copied/distributed or hosted elsewhere without the express permission from American Physiological Society.

\section{Quantitative Models of The Rat Pulmonary Arterial Tree Morphometry Applied to Hypoxia-Induced Arterial Remodeling}

\section{Robert C. Molthen}

Medical College of Wisconsin, Marquette University and Zablocki Veterans Affairs Medical Center, Milwaukee, Wisconsin

Kelly L. Karau

Marquette University, Milwaukee, Wisconsin

Christopher A. Dawson

Medical College of Wisconsin, Marquette University and Zablocki Veterans Affairs Medical Center, Milwaukee, Wisconsin 


\section{Abstract}

Little is known about the constituent hemodynamic consequences of structural changes that occur in the pulmonary arteries during the onset and progression of pulmonary arterial remodeling. Many disease processes are known to be responsible for vascular remodeling that leads to pulmonary arterial hypertension, cor pulmonale, and death. Histology has been the primary tool for evaluating pulmonary remodeling, but it does not provide information on intact vascular structure or the vessel mechanical properties. This study is an extension of our previous work in which we developed an alternative imaging technique to evaluate pulmonary arterial structure. The lungs from Sprague-Dawley rats were removed, perfusion analysis was performed on the isolated lungs, and then an X-ray contrast agent was used to fill the arterial network for imaging. The lungs were scanned over a range of intravascular pressures by volumetric micro-computed tomography, and the arterial morphometry was mapped and measured in the reconstructed isotropic volumes. A quantitative assessment of hemodynamic, structural, and biomechanical differences between rats exposed for 21 days to hypoxia $\left(10 \% \mathrm{O}_{2}\right)$ or normoxia $\left(21.0 \% \mathrm{O}_{2}\right)$ was performed. One metric, the normalized distensibility of the arteries, is significantly $(P<0.001)$ larger $\left[0.025 \pm 0.0011(\mathrm{SE}) \mathrm{mmHg}^{-1}\right](n=9)$ in normoxic rats compared with hypoxic $[0.015 \pm 0.00077$ (SE) $\left.\mathrm{mmHg}^{-1}\right](n=9)$. The results of the study show that these models can be applied to the Sprague-Dawley rat data and, specifically, can be used to differentiate between the hypoxic and the control groups.

There are limited data on the hemodynamic consequences of structural remodeling that occurs in the pulmonary arteries in the presence of pulmonary vascular disease. Idiopathic diseases, such as pulmonary hypertension (PH), cardiovascular diseases, such as aortic stenosis, or chronic lung diseases, such as chronic obstructive pulmonary disease, are known to be responsible for vascular remodeling that often leads to severe pulmonary hypertension, hypertrophy and dilation of the right ventricle, and eventually death. The structural and biomechanical modifications that result from the remodeling then help to perpetuate the remodeling. Understanding the onset and progression of this remodeling process is fundamental to studying the underlying hemodynamic and functional consequences.

The rat has been used as a model of vascular remodeling because it displays significant pulmonary arterial remodeling when exposed to different challenges, such as chronic hypoxia and monocrotaline exposure. Many histological studies of vascular remodeling have been performed in the rat $(7,30,32,37)$, using chronic hypoxia as an experimental stimulus for studying the mechanisms of pulmonary arterial remodeling

$(10,12,16,17,35,36,40)$. The extant luminal morphometry of the pulmonary arterial tree comes almost exclusively from histological measurements performed on plastic corrosion casts $(19,20,42)$, which have been important in developing present concepts regarding normal pulmonary structure-function relationships $(3,6,9)$. Reid and colleagues $(17,37)$ characterized the pattern of pulmonary arterial remodeling induced by hypoxia in rats with histological criteria, including extension of smooth muscle into small, previously nonmuscular arteries, medial thickening in normally muscular arteries, and a decrease in the number of arteries that filled with a hot barium-containing casting material injected at high pressure. Although these changes in the composition of the vessel walls are undoubtedly important, it is the arterial tree geometry and the vessel wall mechanical properties that determine the pulmonary arterial function, and there is little information available as to how these are affected by the remodeling process. The need to expand the characterization of pulmonary arterial remodeling is emphasized by the observation that, within the normal pulmonary arterial tree, the vessel wall histology varies substantially with vessel size $(17,38)$, whereas the passive mechanical properties of the vessel walls appear to be virtually vessel size independent $(1,22)$. In addition, the pulmonary arterial remodeling that occurs in these rats in response to chronic hypoxia appears to result in vessel wall mechanical properties that tend to retain this size independence. 
An immense amount of data analysis and tabulation is required to fully describe the intact arterial tree.

Numerous approaches have been examined for summarizing complex branching networks $(8,9,15,19,20)$. This present work applies a model based on the concept that the pulmonary arterial tree displays a self-consistent structural pattern $(13,14)$ and exploits its recursive structural properties to reduce the measurements to only the main arterial trunk and the first segment in each daughter branch. Therefore, a self-consistent tree can be defined as one in which all subunits (subtrees) downstream from a segment with a given diameter are similar, at least in a statistical sense. Data reduction methods of this type were shown to be plausible in an earlier study of pulmonary arterial morphometry in the rat by Karau et al. (21). One significant benefit of this approach, however, is that the data set still holds requisite hemodynamic information for analysis of arterial flow.

The primary aim of the present study was to apply methods of imaging morphometry to differentiating the pulmonary arterial trees of control rats from rats with pulmonary vascular remodeling secondary to chronic hypoxic exposure. To accomplish this goal, we further develop and analyze methods for representing the morphology of the pulmonary arterial tree. We use measurements made in micro-computed tomography (CT) scans of Sprague-Dawley (SD) rat pulmonary arterial trees and previously developed $(21,22)$ models of morphometry 1) to verify that their branching structure conforms to properties of self-consistency, 2) to examine two methods for estimating the distensibility of the pulmonary arterial tree, 3) to use principles of selfconsistency to introduce a method for comparing data from the main pulmonary arterial trunk to data from minor subtrunk pathways, and 4) to put forward a morphometric vector summary of the pulmonary arterial tree.

\section{METHODS}

\section{Animal exposures.}

To study the intact structure and function of the pulmonary arterial trees, one group of SD rats $(n=9) 55-65$ days old (250-300 g) was exposed to hypoxia by use of an environmental chamber comprised of an airtight acrylic enclosure large enough to house a standard rat cage. The unit has multiple ports through which to regulate inflow and exhaust gases. To produce isobaric hypoxia, a mixture of room air and nitrogen was pumped through the chamber to maintain an oxygen concentration of $10 \%$. Total flow delivery to the chamber was 3-4 $\mathrm{I} / \mathrm{min}$. The chamber was opened daily for several minutes to perform cage, food, water, and bedding replacement and refilling. The rats were placed in the chamber in groups of three to minimize overcrowding and reduce carbon dioxide levels that on average were at $0.45 \%$. The rats were allowed to eat and drink ad libitum. The second group $(n=9)$ was housed under similar but normoxic conditions.

\section{Preparation of the lungs.}

After 21 days, each rat was anesthetized with pentobarbital sodium ( $40 \mathrm{mg} / \mathrm{kg}$ ip), and a midline sternotomy was performed. The rat was heparinized $(200 \mathrm{IU} / \mathrm{kg}$ ) by right ventricular injection, and a blood sample was removed for hematocrit determination. The trachea and pulmonary artery were cannulated, and the heart was excised for right ventricular free wall and left ventricle plus septum weight determination. The lung was ventilated with a $15 \% \mathrm{O}_{2}, 6 \% \mathrm{CO}_{2}$, balance nitrogen mixture, $3 \mathrm{mmHg}$ end-expiratory pressure, and $8 \mathrm{mmHg}$ endinspiratory pressure. The lung was subjected to two or three brief peak inspiratory episodes of $12-15 \mathrm{mmHg}$ to eliminate any atelectasis that might have occurred during the excision $(5,22,43)$. The lung was rinsed free of blood and perfused with a $37^{\circ} \mathrm{C}$ physiological salt solution containing $5 \%$ bovine serum albumin, then a recirculation circuit was created and $6 \mathrm{mg}$ of papaverine in solution were added to the perfusate circulated at 10 $\mathrm{ml} / \mathrm{min}$ for $\sim 2 \mathrm{~min}$. The arterial pressure-vs.-flow relationship was measured over a range from 0 to $40 \mathrm{ml} / \mathrm{min}$, and airway pressure was held constant at $6 \mathrm{mmHg}$. The lung was immediately placed in the imaging chamber and ventilated with the same gas mixture. Using a 10-ml syringe body with the plunger removed as a reservoir for perfluorooctyl bromide (PFOB), we connected one end of the syringe to the pulmonary artery cannula via a 
clamped 1/8 ID Tygon tubing and left the large end of the syringe open to the atmosphere. The physiological salt solution in the lung was then replaced by PFOB in the reservoir by unclamping the Tygon tubing. PFOB does not pass through the capillary bed and therefore only fills the pulmonary arterial tree. Accounting for the density of the PFOB, intravascular pressure was determined by measuring the difference between the height of the surface of PFOB in the reservoir and the center of the lung. The arteries were conditioned by cycling the intravascular pressure from 0 to $30 \mathrm{mmHg}$ several times. As the intravascular pressure is reduced, excess PFOB returns to the reservoir. Ventilation was halted during imaging, and airway pressure was again maintained constant at 6 $\mathrm{mmHg}$. The arterial pressure was set to $30 \mathrm{mmHg}$, and the lungs were rotated in the X-ray beam at $1^{\circ}$ increments to obtain 360 planar images. The pressure was lowered successively to 21,12 , and $5 \mathrm{mmHg}$, with complete CT scans obtained at each pressure (see Fig. 1). Each scan took $\sim 3 \mathrm{~min}$. After imaging, the lungs were drained of PFOB, dried in an oven for at least $2 \mathrm{wk}$, and then weighed.

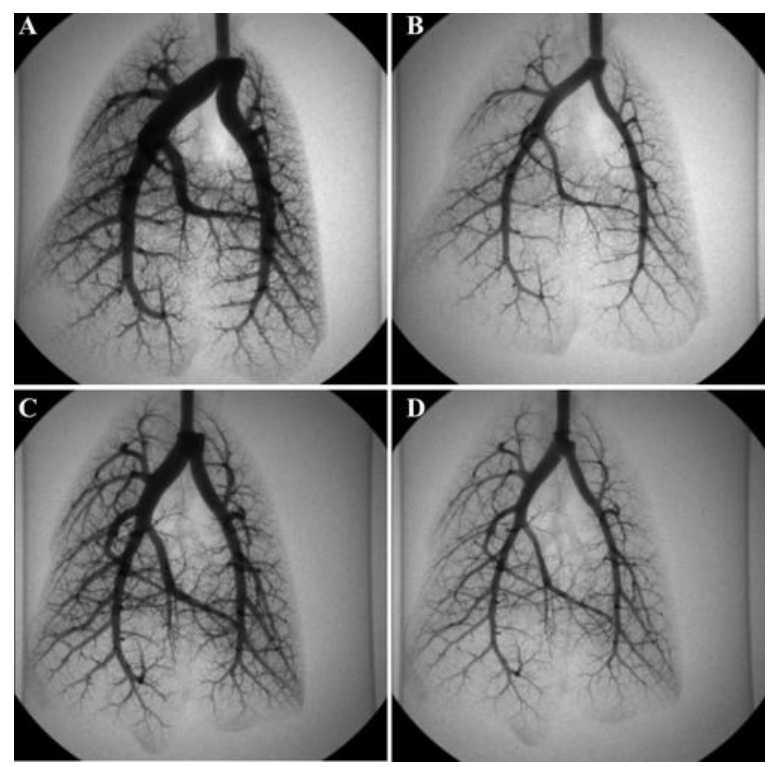

Fig. 1.Planar X-ray projection images of Sprague-Dawley (SD) pulmonary arterial trees filled with perfluorooctyl bromide for contrast. Control $(A$ and $B)$ and hypoxic $(C$ and $D)$ lungs with intra-arterial pressure of $30 \mathrm{mmHg}$ $(A$ and $C)$ and $5 \mathrm{mmHg}(B$ and $D)$.

\section{Imaging.}

The methods for CT imaging and data analysis are similar to those previously described (22). Briefly, the microfocal X-ray CT system is composed of a Fein-Focus-100.50 X-ray source (3- $\mu \mathrm{m}$ focal spot), a North American Imaging Al-5830-HP image intensifier coupled to a Silicon Mountain Design charge-coupled device (CCD) camera, and a New England Affiliated Technologies specimen micromanipulator stage mounted on a precision rail, with position information provided by Mitutoyo linear encoders. Each planar projection image is a result of averaging seven frames to minimize noise as well as maximize vascular contrast. The image data are transmitted from the CCD camera to a frame-grabber board mounted in a Dell 610 workstation running the WindowsNT operating system. Image acquisition, positional information recording, and stage control are all preformed by in-house, custom-written, Windows-based software running on the workstation. The data are then transferred via network to a Linux-based Dell 410 workstation. After proper preprocessing of the projection images to compensate for distortions introduced by the imaging chain (22), isotropic reconstructions are obtained through an implementation of the Feldkamp cone-beam algorithm (11). Figure 2, $A$ and $B$, shows a surface shaded rendering made from $\mathrm{CT}$ data acquired from a normoxic rat arterial tree and its corresponding principal pathway. Figure $2 C$ presents a top view of a similar tree revealing the lateral structure of the network. 

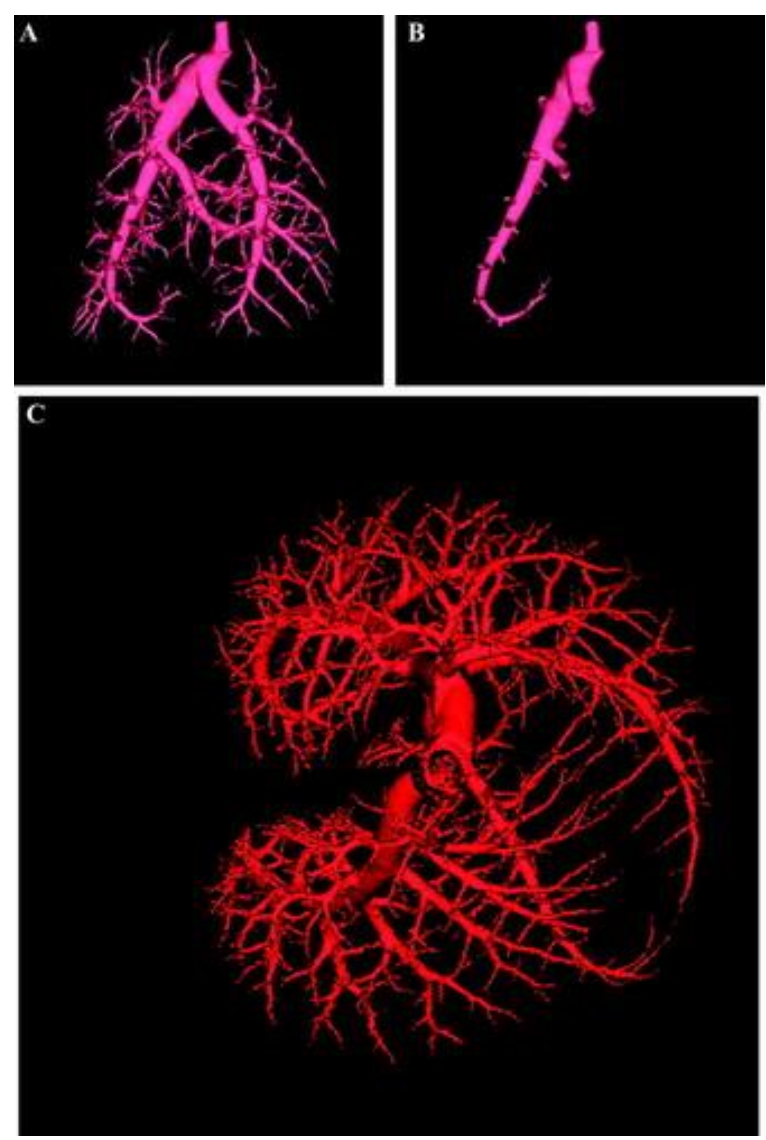

Fig. 2. Thresholded surface-shaded rendering of a cone-beam micro-focal X-ray computed tomography (CT) of a control SD rat pulmonary arterial tree $(A)$ and the corresponding principal pathway $(B)$ and a top view of a similar lung $(C)$.

\section{Morphological measurements.}

The luminal diameter, segment lengths, and the number of branches in the pulmonary arterial tree are key measurement parameters $(15,18,33,39)$ and are available through CT imaging of contrast-filled vessels. Morphometric measurements along pulmonary arterial pathways are made on the isotropic volume data by methods introduced earlier $(21,22)$. Briefly, a modified Gaussian model that takes into consideration the point spread function of the imaging and reconstruction chain was fit to the grayscale CT data for estimation of luminal diameters in the principal pathway. Diacritical coordinates in three dimensions (3-D) were mapped at defined post- and prebifurcation locations throughout the arterial tree. A two-dimensional (2-D) schematic of the principal pathway is shown in Fig. 2B. Circular points indicate the anatomic landmarks along the vessel's central axis at which 3-D coordinates were recorded, and thick gray lines indicate the locations at which crosssectional measurements of luminal diameter were made in arteries ranging in size from $\sim 70$ to $2,800 \mu \mathrm{m}$. Multiple pathway analysis, which was performed on one lung from each group, required mapping and measuring of anatomical points, at locations similar to those shown for the main trunk in Fig. 3, for 10 pathways in the arterial tree of both rats. Although Fig. 3 schematically represents conventional dichotomous branching, the mapping technique accounts for branches coming off the main pulmonary trunk at all angles. Therefore supernumerary vessels branching at approximately right angles from main trunk are included in the morphometric data. Orientation of the plane used for measurement was initially approximated by calculating the plane normal to and midway between the consecutive postbifurcation coordinates. After calculating the estimate, a program written in MATLAB (MathWorks, Natick, MA) enabled the user to visualize and finely manipulate the measurement plane with respect to the artery. The plane was positioned such that, first, it was 
perpendicular to the central axis of the vessel, and second, the 2-D cross section of the lumen revealed the circular structure characteristic of the pulmonary arteries.

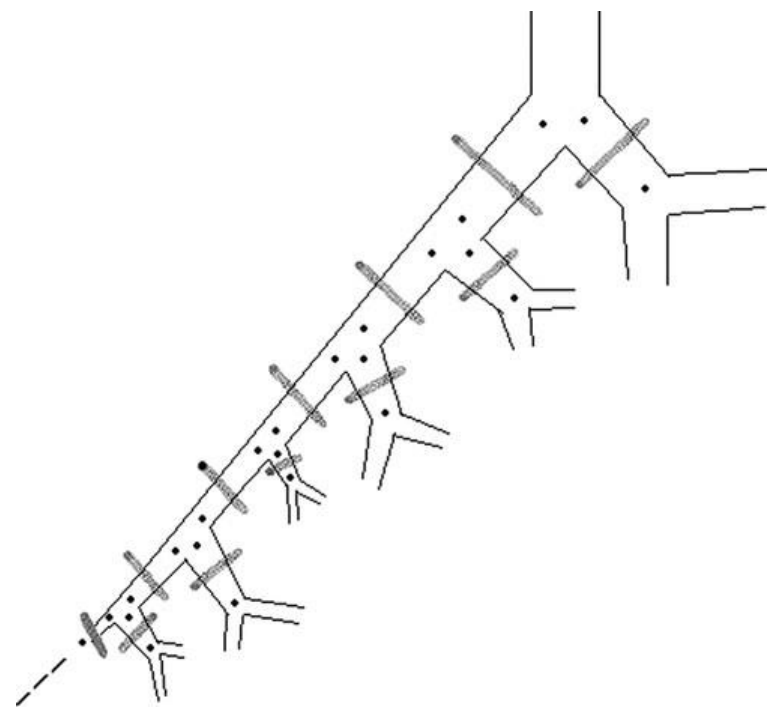

Fig. 3.Schematic 2-dimensional representation of a portion the principal pathway with the first bifurcation of each daughter branch included for clarity. Points indicate the anatomical locations in the center of the vessel at which 3-dimensional coordinates were recorded in each arterial tree, at all pressures imaged. Thick gray lines reflect locations at which luminal diameter measurements were made.

\section{Arterial distensibility.}

Information on vessel wall mechanical properties is fundamental to this study. To estimate the mechanical properties of the vessel wall, additional measurements of the same lung at several different intravascular pressures were made at identical locations in the principal pathway. By modeling the effect of intravascular pressure on vessel diameter we can estimate vessel distensibility as a characteristic of the arterial tree. The pressure recorded in each individual vessel segment is adjusted precisely according to its height difference with respect to the center of the lung.

\section{Data reduction techniques.}

Performing measurements on data confined to the principal pathway, presented above, provides a scheme by which to reduce, to a reasonable extent, the number of measurements required to provide satisfactory results. The notion of a self-consistent tree holds important implications about how much of the vascular tree must be measured to adequately characterize the structure and function of the vascular network. A tree that satisfies the conditions of self-consistency will display the property that the structure of all branches off the main trunk is statistically indistinguishable from their respective subregions of the main trunk. A respective subregion is defined as the portion of the main trunk distal to a point at which the main trunk diameter equals the inlet diameter of the branch being compared. If this condition is met, the momentous feat of measuring all the vessels in the pulmonary tree is reduced to measuring only the main pulmonary trunk and the inlet diameter of the daughter branches along the main trunk. Therefore, measurement of only the main trunk and the inlets to its daughter branches (the "principal pathway") is justified provided that the assumption based on the selfconsistency property holds true for the pulmonary arterial branching structure. In this context, self-consistency provides a means for summarizing the global morphometric and mechanical properties of the vascular tree from a reduced set of measurements. Self-consistency allows an entire tree structure to be characterized and "rebuilt" from principal pathway data. Evidence of self-consistency in the structure of the pulmonary arterial tree was shown in previous work carried out in Fawn-Hooded (FH) rats, a strain that has a known susceptibility 
to $\mathrm{PH}$ (22). In this study we investigated the SD rat, a common laboratory strain. The data presented below shows that these structural characteristics also hold true for normoxic (control) and hypoxia-adapted SD rat lungs.

Using these reduction techniques, we have developed models of morphometry that quantify the rat pulmonary arterial tree using its principal pathway data. Drawing from self-consistency, these models imply that if the diameter-vs.-distance relationship for the main trunk is described by Eq. 1 (below), then the distance-vs.diameter graph of any branch of the tree is described by Eq. 2 (below).

\section{Studies in left lobes.}

After hemodynamic analysis in four of the control lungs, the right lobar artery was ligated and the lobes distal to it were removed. Only the left lung lobe was imaged in these four lungs. Because of the smaller specimen volume, the left lobe could be moved closer to the X-ray source, which provided a gain in the geometric magnification and the resolution of slightly smaller vessels. The left lobes were mapped and measured similarly to full lungs; however, to directly compare morphometrics of left lobes to full lungs, a protocol (presented below) was designed, using properties of self-consistency, to compensate for differences at the primary lobar artery bifurcation. For this reason the left lobe data could be used to calculate the distensibility of the arteries through the individual segment method (also presented below) but not from the surface fit method.

\section{Statistics analysis.}

Data are expressed as means \pm SE. The level of statistical significance was defined as $P \leq 0.001$ for each group of data unless otherwise specified. Student's $t$-test and one- and two-way analyses of variance for multiple responses were performed.

\section{Animal care.}

All protocols were performed according to Institutional Animal Care and Use Committee and conformed to the "Guide for the Care and Use of Laboratory Animals" of the National Institutes of Health.

\section{RESULTS}

\section{Hemodynamics, weights, and hematocrit.}

The underlying difference in the hemodynamic status of the two experimental groups is confirmed by perfusion analysis, performed on the isolated rat lungs. Pulmonary arterial hypertension, reflected by the increase in perfusion pressure or high vascular resistance, is evident and statistically significant in the hypoxia-adapted rats compared with the normoxic controls, as shown in Fig. 4. Hematocrit in the hypoxic group was significantly higher than the controls, $69.1 \pm 1.3$ compared with $41.4 \pm 1.3$. The combination of higher pulmonary vascular resistance and increased viscosity led to an increased workload for the right heart, reflected by a significant amount of right ventricular hypertrophy seen, as measured by the ratio of weight of the dissected right ventricle compared with the remaining left ventricle plus septum, $0.572 \pm 0.043$ in the hypoxic group compared with $0.267 \pm 0.008$ in the control group. By normalizing the right and left heart components to body weight, it appears that there is no general cardiomegaly, because chronic hypoxia caused only the right ventricle to be significantly larger. There was also a significant $(P \leq 0.01)$ increase in dry lung weight in the hypoxic compared with the control group, $0.322 \pm 0.010 \mathrm{~g}$ vs. $0.257 \pm 0.018 \mathrm{~g}$, respectively, both before and after normalizing by body weight. Quantitative morphometric differences between the two groups were also found by analyzing the CT data. 


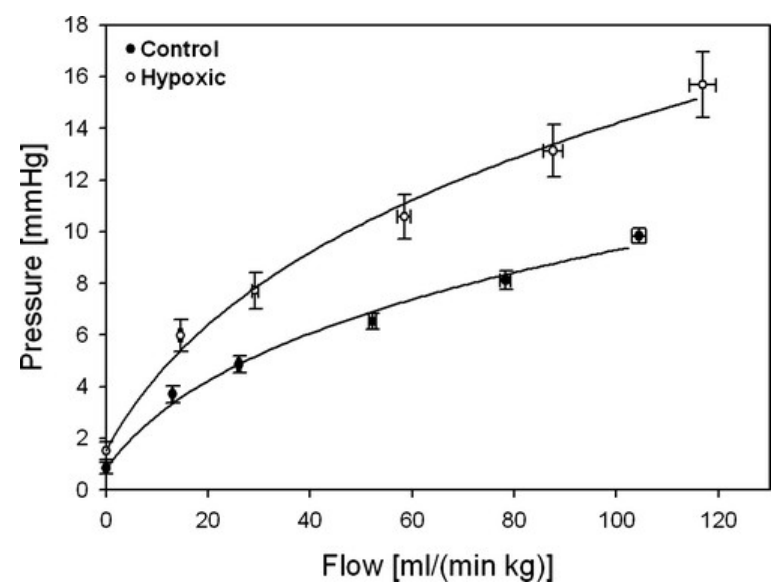

Fig. 4.Pulmonary arterial minus venous pressure vs. flow rate in isolated lungs from normoxic controls $(n=9)$ and hypoxia-adapted $(n=6)$ rats (means \pm SE). Solid lines represent a simple distensible vessel model proposed by Linehan et al. (26). Flow values have been normalized to body weight.

\section{Pulmonary arterial tree morphological models and self-consistency.}

To verify that self-consistent characteristics are present in the pulmonary tree, a semiquantitative analysis was performed on a normal FH rat lung (22). A similar analysis was performed in this study for both an SD rat exposed to hypoxia and an SD control (see Fig. 5). In Karau's previous work, Eq. 1 has been used to describe the relationship between luminal diameter, $D$, and distance along the main trunk, $x$, where $D(0)$ approximates the main trunk diameter at the first bifurcation $(x=0)$, Ltot is the total length of the vessel if it were extended to where the diameter of the vessel equals zero $(D=0)$, and $c$ is a measure of the taper of the vessel from inlet to termination, i.e., when $c<1$ the curvature of the taper is convex to the trunk axis and when $c>1$, the curvature of the taper is concave. Similarly, Eq. 3 has been used to describe the relationship between the cumulative number of branches, $N_{\mathrm{Br}}$, and distance along the main trunk, where $N$ tot is an estimate of the total number of branches off the main trunk and $b$ is a measure of how the branches are distributed along the vessel, i.e., when $b>1$, the number of branches per unit length increases with $x$ and the opposite occurs when $b<1$. 


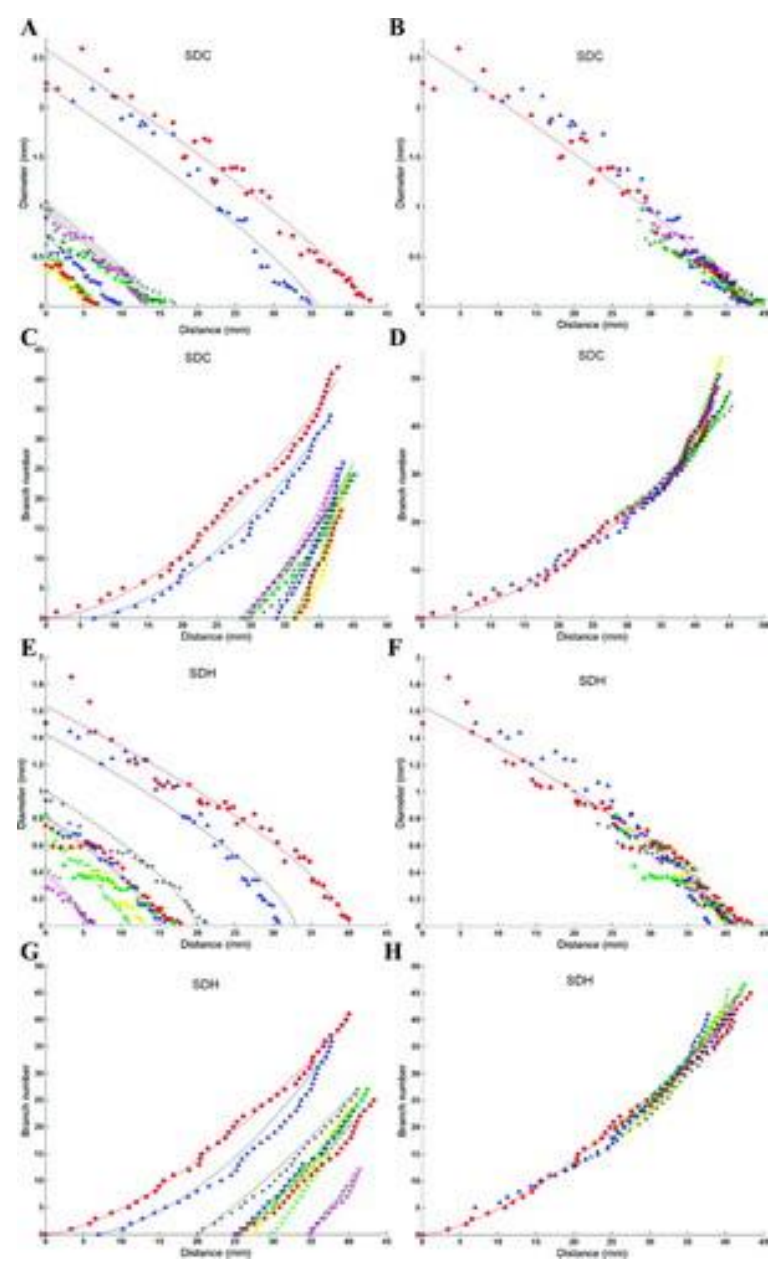

Fig. 5.Data for a Sprague-Dawley control (SDC) lung $(A-D)$ and hypoxic (SDH) lung $(E-H)$. $A$, $E$ : pulmonary arterial diameter vs. distance from the location of the first measurement for the main trunk and 9 minor subtrunk pathways in the example rat lungs. For each pathway the line represents a multi parameter fit to Eq. 1. B, $F$ : the same data for each lung plotted after shifting the data to along the main trunk such that $s_{i}$ in Eq. 2 is found. $C, G$ : cumulative number of branches vs. distance for the same 10 pathways plotted in $A, B, E$, and $F$. Lines represent the model, Eq. 3, fit to the data. $D, H$ : plot of the branch number data, shifted along the main trunk by $n_{i}$ in Eq. 4 .

For both rats studied, two relationships that imply self-consistency were tested. Briefly, the first relationship analyzes the characteristics of the arterial trees that allow the diameter vs. distance along the pathway to be described equally as well by Eq. 1 as by Eq. 2 for any pathway in the tree, where $s_{i}$ indicates the distance the individual subtrunk would need to be shifted along the main trunk to match the equivalent distal portion of the main trunk. Second, the characteristics that allow the cumulative number of branches in a particular pathway vs. distance along the pathway to be described equally as well by Eq. 3 as by Eq. 4, where $n_{i}$ indicates the number of branches down the main trunk the subtrunk had to be shifted. For both relationships $D(0), L$ tot, $b$, and $c$ would be the same for all pathways. For the first test, shown in Fig. 5, $A, B, E$, and $F$, we plot the diameter of 10 vessel trunks vs. the distance from the first measurement along each vessel using the model

$D(x)=D(0)(1-x / L \text { tot })^{c}(1)$

The self-consistent structure of the tree is evident in Fig. 5, $B$ and $F$, where the subtrunks have been appropriately shifted and fit to the model

$D(x)=D(0)\left[1-\left(x+s_{i}\right) / L \text { tot }\right]^{c}(2)$ 
where $s_{i}$ represents the shift for each of the ith subtrunks along the main trunk. Therefore, shifting any of the pathways along the main trunk to their proper position results in a superposition of the pathways. The second test for self-consistency, that the number of branches off any vessel trunk should be similarly distributed, is presented in Fig. 5, $C, D, G$, and $H$. This relationship can be represented by the functional form

$N_{\mathrm{Br}}(x)=N \operatorname{tot}(1-[1-(x / \text { Ltot })])^{\mathrm{b}}(3)$

Congruent with the argument above, the self-consistent tree will display simple shifts of the branch data along the main trunk. This shifting, seen in Fig. 5, $D$ and $H$, would take the form of

$N_{\mathrm{Br}}(x)=N$ tot $\{1-[1-(x / L \text { tot })]\}^{\mathrm{b}}-\eta_{i}(4)$

where in this case $n_{i}$ is the number of branches down the main trunk a vessel must be shifted to match the equivalent portion of the main trunk for each of the ith subtrunks. Inspection of Fig. 5 indicates that selfconsistency conditions are reasonable. Therefore, we conclude, only the morphometrics of the principal pathway need to be measured for a full tree characterization. Using the branch number model, we can determine morphometric characteristics of individual lungs by fitting the measured data to the functional form of Eq. 3. In this manner, data from the main trunk of an arterial tree can be fit simultaneously for the four intravascular pressures studied to determine $N$ tot, $L$ tot, and $b$.

In addition to Eqs. 1 and 3, which are used to represent the main pulmonary trunk, we model the daughter branches by introducing the equation

$D_{\mathrm{Br}}(x)=D_{\mathrm{Br}}(0)(1-x / \text { Ltot })^{C_{\mathrm{Br}}}+\phi_{\mathrm{i}}(5)$

where $D_{\mathrm{Br}}(0)$ is the estimate of the diameter of the first daughter branch off the main trunk, i.e., the left pulmonary artery, and $\phi$ is a variable diameter increment representing the size heterogeneity of the branch diameters. In this case the parameter $c_{\mathrm{Br}}$, unlike $c$, is no longer a description of contour of the taper of the main trunk, but rather it is an attribute of the general trend of the distribution of daughter branch diameters as a function of distance along the main trunk. An appropriate way to model the parameter $\phi$ is to let it equal the coefficient of variation $\left(\mathrm{CV}_{\mathrm{Br}}\right)$ obtained from the variance in the measurement of the branch diameters around the fitted Eqs. 1 and 5, using the five free parameters $D(0), D_{\mathrm{Br}}(0)$, Ltot, $c$, and $c_{\mathrm{Br}}$, with $\phi=0$.

$\mathrm{CV}_{\mathrm{Br}}=\sqrt{\frac{\left(\frac{D_{\mathrm{Br}} \text { data- }-D_{\mathrm{Br}} \mathrm{fit}}{D_{\mathrm{Br}} \mathrm{fit}}\right)-\left(\frac{\text { Ddata-D fit }}{D \text { fit }}\right)}{N-5}}(6)$

where "data" refers to the measured value and "fit" refers to the value predicted by Eqs. 1 and 5. Here CV is a relative index used to gain an appreciation for the size heterogeneity in the branches off the main trunk.

Therefore, a reduced set, graphical characterization of any given lung can be represented by a graph analogous to those shown in Fig. 6, where the main trunk and the daughter branches have been fit by the appropriate functional model equations, and $D(0), L$ tot, and $c$ are free parameters, although $L$ tot was limited to a value greater than or equal to the length of the measured pathway. It is important to note that although Eq. 5 is a continuous function, the physical relevance only is applicable at the $N$ tot discrete locations along the main trunk where the daughter branches actually exist. 

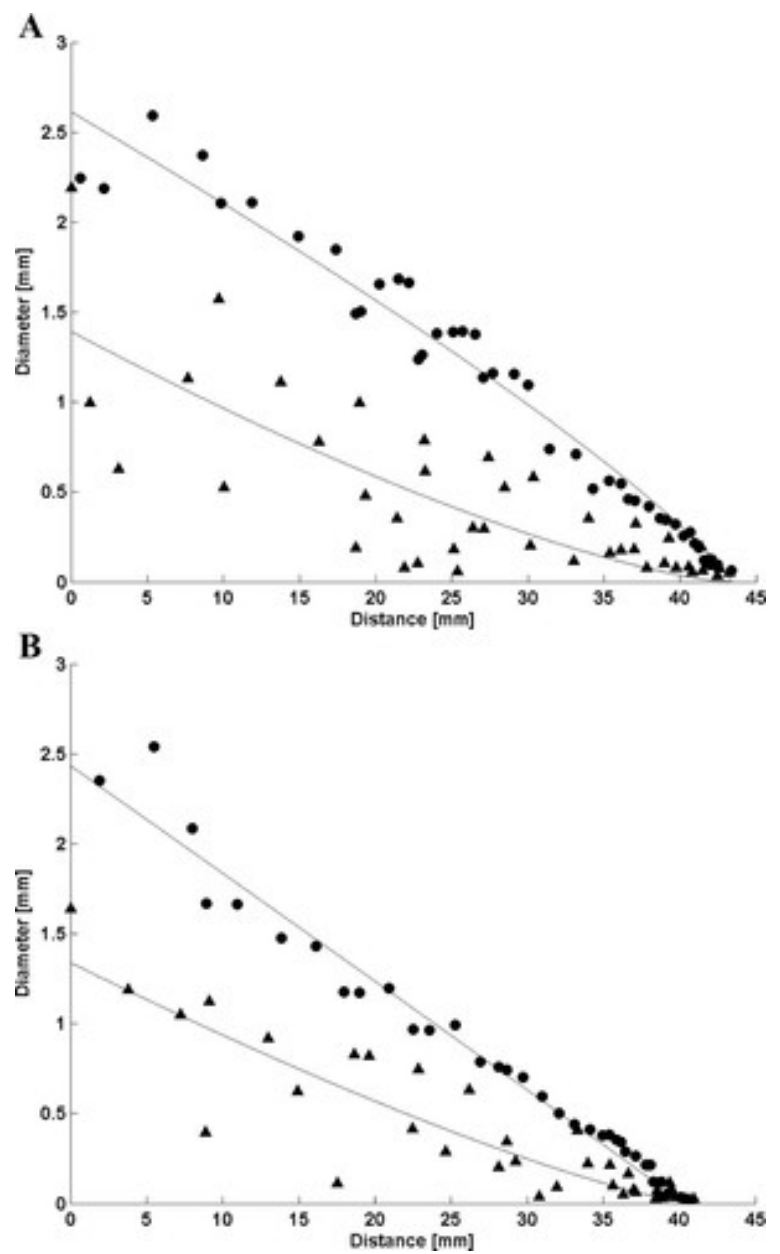

Fig. 6. Principal pathway data for an example SDC $(A)$ and SDH $(B)$ rat lung at an intravascular pressure of 30 $\mathrm{mmHg}$. Data points represent diameters vs. distance from inlet for principal path segments. $\bullet$, main trunk data; $\triangle$, daughter branches. Lines represent the model fit to the data where the main trunk data are fit to Eq. 1 and the branch data fit to Eq. 5 with $\phi=0$.

\section{Arterial distensibility.}

To better understand how the principal pathway behaves under the influence of different intravascular pressures, we have also developed models that incorporate the distensibility of pulmonary arteries. We wish to describe the arterial distensibility behavior over a range of pressures as one quantitative measure. We can express the distance-vs.-diameter relationship as a function of pressure by incorporating the well-described $(1,41,44)$ relationship between diameter and pressure

$D(x, \mathrm{P})=D(x, 0)(1+\alpha \mathrm{P})(7)$

where $\alpha$ is a distensibility coefficient relating a fractional change in diameter per unit change in pressure with respect to the unstressed vessel diameter, $D(x, P=0)$. This pressure dependence can be consolidated into Eqs. 1 and 5 in the following forms

$D(x, \boldsymbol{P})=D(0,0)(1+\alpha \mathrm{P})\left(1-x /\right.$ Ltot $^{c}(8)$

and

$D(x, \mathrm{P})=D_{\mathrm{Br}}(0,0)(1+\alpha \mathrm{P})\left(1-x /\right.$ Ltot $^{{ }^{\mathrm{B}}}{ }^{\mathrm{Br}}(9)$ 
Therefore analogous to the above development for a single pressure, a reduced-set, graphical characterization of the biomechanical properties of a given lung can be represented by the graphs shown in Fig. 7, where the fitted surfaces represent the vessel behavior over the range of experimental pressures and the tilt of the surfaces reflects the distensibility of the vessels. To estimate the global value of $\alpha$ for a lung, both the main trunk and daughter branches are initially fit for the free parameters of $D(0), L$ tot, $c$, and $\alpha$ values, and then Eqs. 8 and 9 are simultaneously fit only for the free parameter $\alpha$. Figure 8 shows the $\alpha$ values estimated for the individual components of the principal pathway compared with the value generated from a global fit for the entire principal pathway. The difference in $\alpha$ was significant for each group of measurements.
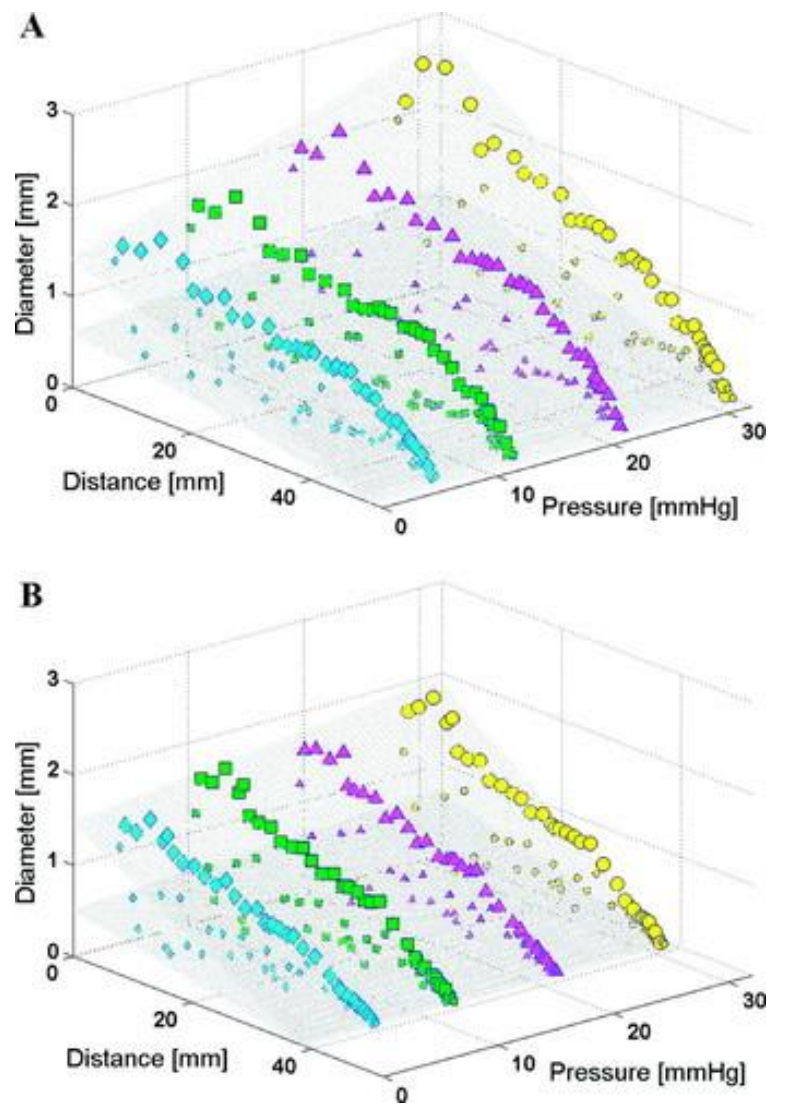

Fig. 7.Principal pathway data for an example control $(A)$ and hypoxic $(B)$ rat lung plotted for the 4 intravascular pressures studies, $\downarrow, 5 \mathrm{mmHg}$;, $12 \mathrm{mmHg}$; , $21 \mathrm{mmHg} ; \bullet, 30 \mathrm{mmHg}$. Data points represent diameters vs. distance from inlet for principal path segments. Large markers, main trunk data; small markers, branch data. Surfaces represent the model fits to the principal pathway data. The tilt of the surfaces is a reflection of the arterial distensibility.

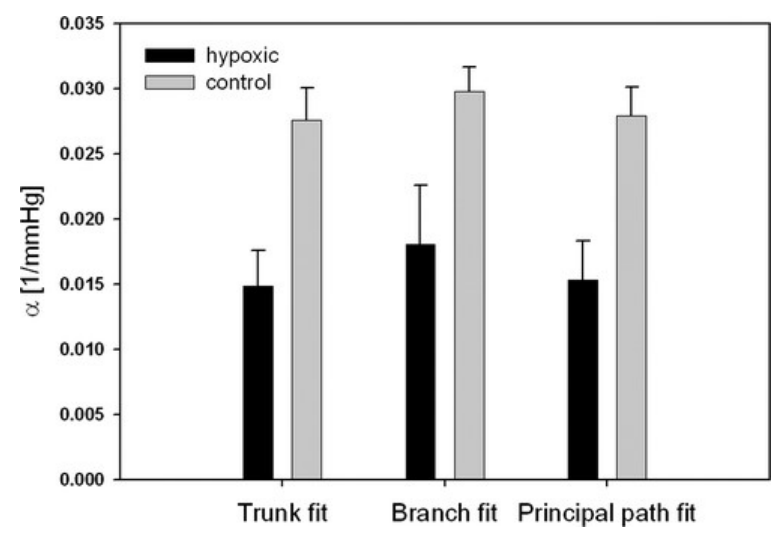


Fig. 8. $\alpha$ (Mean $\pm S E$ ) values estimated from a surface fit to the main trunk data, the daughter branch data, and the principal pathway data (both) for hypoxic $(n=8)$ and control $(n=5)$ SD rat lungs.

Thus one way to estimate the distensibility of the vessel walls is to simultaneously fit the main trunk and daughter branch data to the functional forms for all experimental pressures to estimate the value of $\alpha$. Alternatively, one could use the linear pressure vs. diameter relationship proposed by Yen et al. (41), which is essentially true over the range of pressures investigated in this study, that is

$D(\mathrm{P})=\beta \mathrm{P}+D(0)(10)$

where $\beta$ is a related distensibility coefficient for the individual vessel segments. We can then calculate $\alpha$ from the normalized relationship

$\alpha=\beta / D(0)(11)$

for estimating the global mechanical properties of the vessel walls. This method uses measurements made on each vessel segment throughout the principal pathway at all the intravascular pressures at which data was acquired regardless of the segments position in the arterial tree. For example, each line in Fig. 9 represents linear regression performed on an individual vessel segment, where both the unstressed diameter, $D(0)$, and the segment's $\beta$ value are estimated.

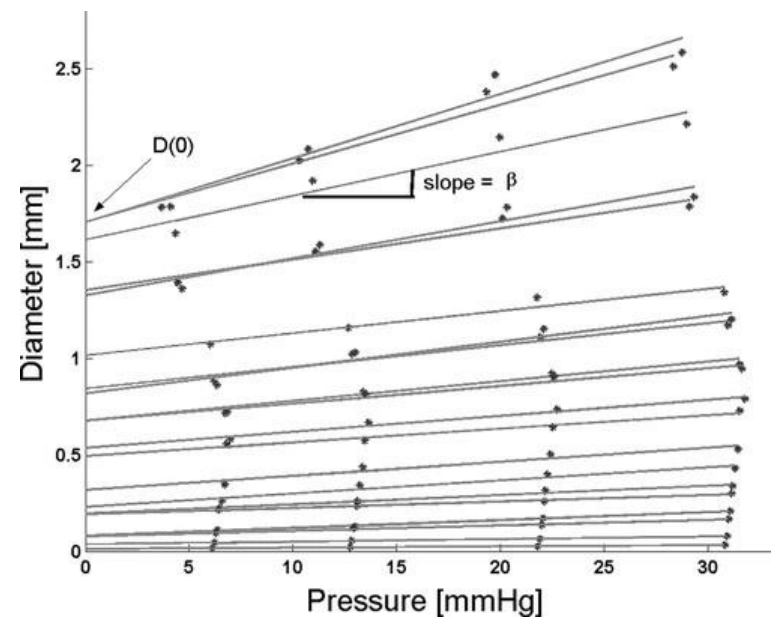

Fig. 9.Diameter vs. pressure of 21 example pulmonary arterial vessel segments from a hypoxic rat lung plotted for the range of intravascular pressures $(*)$. Lines represent the linear regression for each segment. The slope of the line is an local estimate of vessel elasticity, $\beta$, and the $y$-intercept an estimate of the unstressed vessel diameter, $D(0)$.

Examining the relationship between the values of $D(0)$ and $\beta$ for all the vessel segments reveals that the data are well represented by a line, which has an intercept that is not significantly different from zero, as seen in Fig. 10. To confirm there was no significant difference between the standard regression line and the regression line forced to have a zero intercept, an F-test was performed on all of the data sets, according to methods proposed by Motulsky and Ransnas (34). Because the regression line effectively passes through zero, we conclude that the distensibilities of the vessels throughout the tree are diameter independent and, thus, it conditionally allows us to use the estimated slope of this line as a global representation of the tree's distensibility $\alpha$. The mean estimated values ( \pm SE) of $\alpha$ in both groups for both the surface fit method (full lung data used) and the individual distensibility method for determining $\alpha$, as described above, are given in Table 1. 


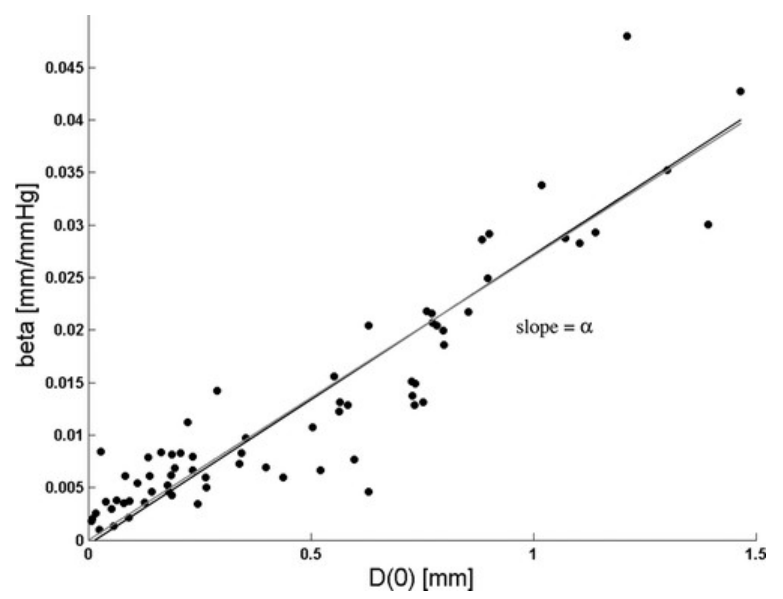

Fig. 10. $\beta$ Vs. $D(0)$ plotted for all artery segments in the principal pathway of an example control SD rat lung. One line represents the linear regression of the data; the second line is the regression with the intercept forced through zero. The difficulty in seeing the 2 individual lines reflects the insignificant difference between them. The slope of the zero intercept relationship is used to estimate $\alpha$.

Table 1. Values of $\alpha$ estimated from surface fit and individual vessel distensibility methods for various hypoxic and normoxic Sprague-Dowley rats

\begin{tabular}{|l|l|l|}
\hline & Hypoxic & Control \\
\hline Surface fit & $0.015 \pm 0.003(n=8)$ & $0.028 \pm 0.001(n=5)$ \\
\hline Individual segments & $0.015 \pm 0.001(n=9)$ & $0.025 \pm 0.001(n=9)$ \\
\hline
\end{tabular}

Values are means $\pm \mathrm{SE}$, in $\mathrm{mmHg}^{-1}$.

\section{Equivalent lobes.}

One goal of this work is to develop a parameter set or vector that is capable of summarizing the tree morphometry including mechanical behavior. To compare morphometry in the intact full tree to left lobe data acquired, a technique was implemented to convert full lung principal pathway data into its equivalent selfconsistent lobe. Briefly, the main trunk data from the full lung at $30 \mathrm{mmHg}$ was first fit to $\mathrm{Eq}$. 1 . Then the diameter in the model equal to the diameter of the first daughter branch was identified. The location along the pathway corresponding to this model diameter was used to determine the equivalent lobe inlets (see Fig. 11). The inlet segment for each pressure data set and corresponding model was chosen to be the first trunk segment upstream from the offset point. The main trunk and corresponding daughter branch data distal to and including each inlet segment were defined as the equivalent lobe. 

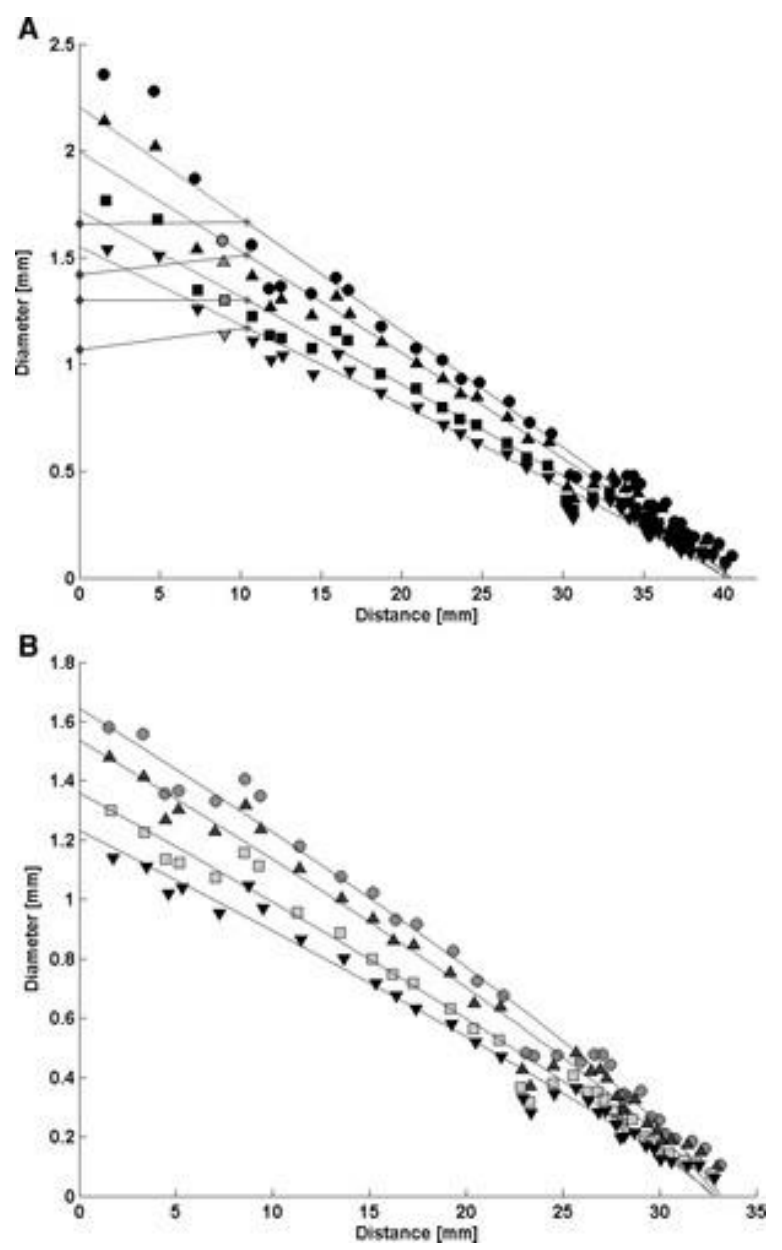

Fig. 11.Creating a simulated lobe from full lung data. $A$ : main trunk data from an example SDH lung for the 4 intravascular pressures imaged, $\bullet, 5 \mathrm{mmHg} ; \cdot, 12 \mathrm{mmHg} ; \bullet, 21 \mathrm{mmHg} ; \bullet, 30 \mathrm{mmHg}$. A shaded marker in each pressure data set indicates the inlet segment chosen for the simulated lobe in $B$. $\downarrow$, First daughter branch at each pressure; ${ }^{*}$, points on the model fits used to choose the simulated lobe inlet. $B$ : equivalent lobe created from all data distal to the chosen inlet segment is refit to a main trunk model. Corresponding daughter branch data are included in the equivalent lobe but not shown for clarity.

\section{Morphometric parameter summary.}

Using a combination of the models above, we can use a morphometric vector or parameter set including no. of segments, $D(0,0), \mathrm{E}_{\mathrm{T}}, \mathrm{D}_{\mathrm{Br}}(0,0)$, Ltot, Ntot, $c, c_{\mathrm{Br}}, b, \alpha, \mathrm{CV}_{\mathrm{Br}}$ to describe the morphometry of each tree. $\mathrm{E}_{\mathrm{T}}$ is the sum of the squared errors between the main trunk data and the model. Table 2 presents values (means $\pm \mathrm{SE}$ ) calculated for the two experimental groups, for full lung data and for the left lobe and equivalent lobe data. The overall morphology of control and hypoxic lungs is similar. However, in addition to the significant difference in the $\alpha$ parameter, the trend that there is a better fit of the main trunk data to the model for the full hypoxic lungs compared with the full control lungs becomes significant $(P<0.005)$ for the equivalent lobes.

Table 2. Morphometric vectors

\begin{tabular}{|l|l|l|l|l|l|}
\hline & & Full Lung & & Equivalent Lobes & \\
\hline & & Control $(n=5)$ & Hypoxic $(n=8)$ & Control $(n=9)$ & Hypoxic $(n=8)$ \\
\hline No. of segments & $39.000 \pm 1.643$ & $37.250 \pm 1.098$ & $34.222 \pm 1.498$ & $32.625 \pm 1.700$ & \\
\hline$D(0,0)$ & $1.536 \pm 0.033$ & $1.563 \pm 0.062$ & $1.343 \pm 0.057$ & $1.243 \pm 0.064$ & \\
\hline Trunk fit SSE & $1.760 \pm 0.334$ & $1.228 \pm 0.086$ & $1.847^{*} \pm 0.322$ & $0.615 \pm 0.108$ & \\
\hline
\end{tabular}




\begin{tabular}{|l|l|l|l|l|l|}
\hline$D_{\mathrm{Br}}(0,0)$ & $0.763 \pm 0.031$ & $0.755 \pm 0.060$ & $0.521 \pm 0.029$ & $0.501 \pm 0.046$ & \\
\hline$L_{\text {tot }}$ & $44.086^{*} \pm 1.512$ & $39.861 \pm 0.643$ & $32.523 \pm 1.077$ & $32.158 \pm 1.202$ & \\
\hline$N$ tot & $36.314^{*} \pm 2.617$ & $28.992 \pm 1.024$ & $27.647 \pm 2.426$ & $29.771 \pm 2.282$ & \\
\hline $\boldsymbol{C}$ & $0.935 \pm 0.043$ & $0.938 \pm 0.035$ & $0.817 \pm 0.036$ & $0.895 \pm 0.025$ & \\
\hline$C_{\mathrm{Br}}$ & $1.409 \pm 0.093$ & $1.393 \pm 0.103$ & $0.991 \pm 0.124$ & $1.002 \pm 0.073$ & \\
\hline$b$ & $1.845 \pm 0.117$ & $1.782 \pm 0.097$ & $1.929 \pm 0.158$ & $1.694 \pm 0.107$ & \\
\hline$\alpha$ & $0.028^{*} \pm 0.001$ & $0.015 \pm 0.001$ & $0.028^{*} \pm 0.001$ & $0.014 \pm 0.002$ & \\
\hline $\mathrm{CV}_{\mathrm{Br}}$ & $0.815 \pm 0.105$ & $2.909 \pm 1.977$ & $0.761 \pm 0.107$ & $0.609 \pm 0.051$ & \\
\hline
\end{tabular}

Various morphology parameters obtained from control and hypoxic rat lungs. Data are shown for full lung and (equivalent) left lobes. Values are means $\pm \mathrm{SE}$. $\mathrm{D}(0,0)$, main trunk luminal diameter at first bifurcation; SSE, sum of squared errors; $\operatorname{DBr}(0,0)$, estimated diameter of first daughter branch; Ltot, total length of the vessel if it were extended to where its diameter equals zero; Ntot, estimate of total number of branches off the main trunk; c, measure of the taper of the vessel from in let to termination; $\mathrm{cBr}$, trend in consecutive daughter branch diameters; $b$, measure of how branches are distributed along the vessel; $\alpha$, distensibility coefficient; $\mathrm{CVBr}$, coefficient of variation of branch diameters.

*Parameters that display a significant difference $(P \leq 0.05)$

\section{DISCUSSION}

The morphometric models presented here are concise representations of the pulmonary arterial tree. The models are based on self-consistency of branching networks, an idealization that allows us to summarize the tree structure and permits efficiency in simulation of model networks. Self-consistency permits simulation of the network's dynamic response while retaining dimensionalities of heterogeneity, complex asymmetrical branching structure, and vessel mechanical properties. The models can be used to describe differences in the pulmonary arterial tree morphometry in rats. In this study, model parameters that estimate arterial distensibility and total length and total number of branches in the main pulmonary arterial trunk differ significantly between rats raised breathing either $10 \% \mathrm{O}_{2}$ for 21 days or room air.

There are fundamental differences between the models presented in this paper and those that represent the pulmonary arterial tree structure as fractal. The criteria used to demonstrate that a structure is self-consistent are less strict than those in fractal models used to verify that a structure is self-similar. Rather than prove that the branching characteristics of the pulmonary arterial tree display similar characteristics at different scales of measurement and consequently have mathematically definable fractal dimensions, we show that the morphology of daughter branches is analogous to a respective portion of the main trunk. Because a selfconsistent tree does not strictly follow fractal scaling rules, the deviations from fractal dimension seen in the first several generations in many biological trees do not necessarily apply as criteria for characterizing differences between trees.

\section{Morphometric models.}

The models proposed in this paper are useful and descriptive. Although concise morphology can be determined from the arterial tree by using the present method of analysis, there are several characteristics of arterial morphology that are not adequately represented. For example, in Figs. 5-7, there are interesting stair-step patterns located in approximately the middle third of the main trunk diameter-vs.-distance relationship. The steps, most evident at the higher intravascular pressures, are almost ubiquitous and characteristically different between the two experimental groups. In the control group, the steps are concave downward, whereas the steps tend to be flat or have a slight upward curvature in the hypoxic rats. Compared with data from hypoxic rats, control data have a larger divergence from the main trunk model, as seen in the parameter "trunk fit SSE" 
in Table 2. This difference is significant in the equivalent lobe data. The stair-steps reflect underlying structure associated with a range of relatively large branches off the main trunk that may coincide with the thick walled oblique muscle segment identified by Meyrick et al. (29) in the range of the 9th and the 20th generation.

In addition, inspection of the planar images reveals subtle differences between the control and hypoxic arterial trees. One important difference is the decrease in "background haze" observed by others $(12,17)$ and also seen in our arteriograms, Fig. 1, of rats exposed to chronic hypoxia. The haze represents small, probably subresolvable vessels that have a large impact on recruitment and consequently hemodynamics. The principal pathway is a set of contiguous segments that reflect the single longest path from the inlet (main pulmonary artery) to the terminal vessel (the smallest measurable artery in our analysis) and defines the minimal data required to represent the tree. The principal pathway analysis does not comprehensively address what may be occurring in many of the parallel distal vessels. Our models did show a significant difference in the morphometric parameters Ntot and Ltot in the chronic hypoxic group; however, when normalized by body weight, the differences were no longer significant. A more comprehensive measurement and parameterization of the distal tree would provide better insight into the changes in the small arterial network.

Another parameter of interest is the branch size heterogeneity. Changes in this parameter may reflect developmental or remodeled structure resulting in differences in flow distribution throughout the main trunk. We have suggested a way to model the parameter $\phi$ in $E q .5$ is to estimate a coefficient of variation $\left(C_{\mathrm{Br}}\right)$, described in Eq. 6, to quantitate the branch size heterogeneity. In this calculation we consider the idea that there is variability in the main trunk data, which could be due to actual distortions dependent on proximity of the measurement plane to a bifurcation or could be due to errors in the diameter measurement. Similarly, this variability exists in the daughter branch measurements. Subtracting the variance about the fit to Eq. 1 from that about Eq. 5 provides some approximation to the variance in branch diameters due to the heterogeneity in the branch-to-trunk ratio. The normality of diameter measurements for each of the first 20 generations of main trunk and daughter branches for hypoxic and control rats was tested individually with a Kolmogorov-Smirnov test with $P \leq 0.05$ and passed. Therefore, estimating the $\mathrm{CV}_{\mathrm{Br}}$ parameter according to $E q .6$ should be a fair measure of a branch heterogeneity index.

The branch diameter heterogeneity is also a reflection of the developmental 3-D, volume-filling nature of the pulmonary vasculature. Consequently there is likely a predictable dependence on branch rotation angle, as evident by observing the top view in Fig. 2 . The models presented here do not include parameterization of the branch rotation angle. Future models that include rotation angle and its dependence on the daughter branching pattern will be important and are very compelling.

We found that a decrease in the pulmonary arterial distensibility measured in rats subject to hypoxic exposure is significant and related to the vascular remodeling that occurs in this group. We calculated a similar value for the pulmonary arterial tree distensibility using both methods presented. Estimating distensibility and other modelfitting procedures in this study require only modest computation time; however, the process of measuring the individual principal pathway segment diameters at each pressure is very time consuming. We are investigating new methods to autonomously detect, measure, and represent the tree more comprehensively and at the same time save resources and increase repeatability.

In addition to identifying differences seen in models of hypoxia, reduced-set morphometric measurements in the intact pulmonary arterial tree can be used to build predictive, analytical models of the pulmonary circulation $(8,23,24)$. These models can provide information regarding flow partitioning, blood volume distribution, and insights into the hemodynamic consequences of remodeling. Our results suggest that a key hemodynamic feature in the PH rat model may be the decreasing distensibility in the pulmonary arteries. Some preliminary work by our group using mathematical hemodynamic models suggests that a change in arterial distensibility 
alone does not account for the pulmonary pressure elevations seen in chronically hypoxic rats (G. S. Krenz, unpublished observations), and further study is required to understand the roles of the individual remodeling components.

\section{Hemodynamics, weights, and hematocrit.}

Changes in the measured hemodynamic and physiological parameters, such as increased pulmonary vascular resistance, right ventricular hypertrophy, increased hematocrit, and a reduction in vessel filling in rats chronically exposed to hypoxic are consistent with the changes reported by other groups $(31,37)$. Although we did not measure pulmonary arterial pressure (PAP) in vivo, we can safely assume that, in addition to the high perfusion pressure noted in Fig. 4, the elevated hematocrit in the hypoxic group would have greatly accentuated the in vivo PAP. Large increases in PAP have been implicated as a "second hit," promoting further vascular remodeling and the development of neointimal lesions (4). Histological examination was not performed on the lung tissue in this study, although the significant increase in the dry lung weight in the hypoxic group suggests that remodeling was present. The remodeling was likely a combination of an increase in arterial wall thickness, extension and hypertrophy of smooth muscle cells, as well as an increase in the extracellular matrix well describe in hypoxic SD rats (17).

\section{Isolated lung preparations.}

The ventilation and perfusion parameters for the isolated lung preparations were chosen to match those used in previous studies $(5,21,37,43)$. They maintain the perfusate $\mathrm{pH}$ close to 7.4 through the bicarbonate buffer system and provide adequate inflation and lung clearance. Papaverine was added to the perfusate to eliminate the effect of active muscle tone. A measure of the parenchymal mechanical status, the lung compliance, has been shown not to change after chronic hypoxic exposure (2) and was not measured in this study. Variations in airway pressure will affect the pulmonary vascular resistance. For example, increasing airway pressure increases the PAP measured in perfusion studies. In our experiments, constant airway pressure of $6 \mathrm{mmHg}$ provided a transpulmonary differential within the physiological range and consistency between hemodynamic and imaging studies.

\section{Vessel measuring methodologies.}

Vascular casting with either barium gel or plastic resins has unquestionably contributed to our understanding of normal pulmonary structure-function relationships $(3,7,9,29)$. Besides the process being arduous, technical difficulties, such as filling the entire arterial network homogeneously, validation of downstream pressures, and measuring the mechanical response to a range of intravascular pressures have dissuaded its use as a tool for characterizing vascular remodeling; consequently, the data obtained to date are from a relatively small number of lungs. In addition, histological observations, which have been the primary tool for evaluating pulmonary vascular remodeling, do not provide information on intact 3-D vascular tree structure or the mechanical properties of the vessels. As an alternative, we have developed a volumetric X-ray micro-CT imaging technique that increases the throughput enough to make such studies more practical. In our preparations, the arterial tree is filled with perfluorooctyl bromide, a very-low-viscosity contrast agent that does not pass through the capillary bed. CT has the key advantages that the resulting 3-D data set is rapidly available in digitized form and comprised of all the vessels with their correct spatial orientation and connectivity; in addition, the data can be collected on the same lung under different experimental conditions, allowing for evaluation of vessel mechanics as well as structure $(22,27)$.

\section{Imaging limitations.}

The resolution limit of the scanner theoretically approaches the system's focal spot size of $3 \mu \mathrm{m}$ but is also impacted by the imaging chain and the reconstruction algorithm. Although the closer the object can be placed to 
the X-ray source, the higher the resolution possible, the smallest vessel measured, $\sim 70 \mu \mathrm{m}$, was effectively limited by the size of the object (the lung) scanned and the requirement that the object remain in the detector (the image intensifier) field for every projection view used to reconstruct the tomographic data. The detector aperture can be set as large as $22 \mathrm{~cm}$ to image larger objects; however, magnification is lost because large objects require the source-to-object and source-to-detector distance to increase, limiting the size of the smallest contrasted vessel that can be resolved and consequently measured. A significant amount of remodeling is known to occur in the partially muscular and nonmuscular arteries with diameters $<100 \mu \mathrm{m}(37)$. Our measurements only sample the upper range of small vessels and do not extend to the capillary level. In addition to not having data for this important portion of the arterial tree, methods for branch ordering, such as Horsfield and Strahler systems $(18,19)$, which require knowledge of the end branches, are not directly applicable.

\section{Genetic and clinical relevance.}

Idiopathic forms of PH have been linked to a mutation in bone morphogenetic protein receptor (BMPR2) $(25,28)$. We also now know the number of genes coding for pulmonary structure is much fewer in number than components that make up the lung, i.e., there is not an individual gene for every capillary, alveolus, and peripheral branch. Earlier we showed (22) that the FH lung displays self-consistency. Here we have shown arterial trees in SD rats are also self-consistent and therefore can assume that, in addition to the vascular structure, the distribution of blood flow in any subtree of the arterial network is also statistically indistinguishable from its respective main trunk subregion. It is then logical that a self-consistent or recursive rule exists at some level that guides arterial development and plays some role in guiding the remodeling process, because, before and after significant remodeling, vessel distensibility remains virtually independent of diameter. The FH rat is known and studied for its susceptibility to pulmonary hypertension. We are presently performing studies to compare the morphometry of the pulmonary arterial tree in different rat strains. The $\mathrm{FH}$ rat has an increased baseline pulmonary vascular resistance, is more sensitive to hypoxic exposure, and develops PH spontaneously. However, estimates of arterial distensibility under control and hypoxia are equivalent in SD and FH strains (R. C. Molthen, unpublished observations). We still suspect that phenotypic structural differences exist between strains that determine underlying factors that cause susceptibility or resistance to $\mathrm{PH}$.

Although the initiation and progression of arterial remodeling is still unclear, we know that a positive feedback control loop is at work in the remodeling process. Genetic predisposition seemingly sets the stage for an increased sensitivity to this counterproductive response. The $\mathrm{FH}$ rat and humans genetically predisposed to $\mathrm{PH}$ may be more sensitive to remodeling stimuli, effectively short-circuiting loops of the feedback algorithm.

3-D volumetric CT is becoming a standard-of-care in the clinical environment and is often ordered for lung and thoracic scans. Methods presented here for estimating pulmonary arterial distensibility are not directly applicable to clinical studies; however, one could envision imaging technology improving to the point that accurate temporal scanning of the pulmonary vasculature could be recorded at different points in the cardiac cycle, i.e., at systole and diastole, such that estimates of pulmonary arterial distensibility could be used to diagnose occult remodeling accompanying PH. A parametric vector-based approach, such as the one presented in this study, would identify quantitative differences between groups; it may also help to diagnose what stage of the process an individual is at and the most effective strategy for treatment at particular stage.

In conclusion, we believe that strong arguments exist for applying CT imaging and 3-D isotropic reconstruction to the problem of measuring arterial morphology and biomechanical measurements. These measurements provide important information about the current state of the pulmonary vessels and how the vessels respond to external stimuli and could supply clues regarding the genetic influences driving the development of pulmonary hypertension and its pathophysiology. 


\section{GRANTS}

This work supported by National Heart, Lung, and Blood Institute Grants HL-19298 and HL-64368, the W. M. Keck Foundation, and the Department of Veterans Affairs.

\section{FOOTNOTES}

- The costs of publication of this article were defrayed in part by the payment of page charges. The article must therefore be hereby marked "advertisement" in accordance with 18 U.S.C. Section 1734 solely to indicate this fact.

The authors thank Amy Heinrich, Gary Krenz, and Steve Haworth.

\section{REFERENCES}

1 Al-Tinawi A, Madden JA, Dawson CA, Linehan JH, Harder DR, and Rickaby DA. Distensibility of small arteries of the dog lung. J Appl Physiol 71: 1714-1722, 1991.

2 Barer GR, Herget J, Sloan PJ, and Suggett AJ. The effect of acute and chronic hypoxia on thoracic gas volume in anaesthetized rats. J Physiol 277: 177-192, 1978.

3 Bennett SH, Goetzman BW, Milstein JM, and Pannu JS. Role of arterial design on pulse wave reflection in a fractal pulmonary network. J Appl Physiol 80: 1033-1056, 1996.

4 Botney MD. Role of hemodynamics in pulmonary vascular remodeling: implications for primary pulmonary hypertension. Am J Respir Crit Care Med 159: 361-364, 1999.

5 Chander A. Regulation of lung surfactant secretion by intracellular pH. Am J Physiol Lung Cell Mol Physiol 257: L354-L360, 1989.

6 Cumming C, Henderson R, Horsfield K, and Singhai S. The functional morphology of the pulmonary circulation. In: The Pulmonary Circulation and Interstitial Space, edited by Fishman AP and Hecht HH. Chicago, IL: Univ. of Chicago Press, 1969, p. 327-340.

7 Davies P, Maddalo F, and Reid L. Effects of chronic hypoxia on structure and reactivity of rat lung microvessels. J Appl Physiol 58: 795-801, 1985.

8 Dawson CA, Krenz GS, Karau KL, Haworth ST, Hanger CC, and Linehan JH. Structure-function relationships in the pulmonary arterial tree. J Appl Physiol 86: 569-583, 1999.

9 Dawson CA, Krenz GS, and Linehan JH. Complexity and structure-function relationships in the pulmonary arterial tree. In: Complexity in Structure and Function of the Lung, edited by Hlastala MP and Robertson HT. New York: Dekker, 1998, p. 401-427. (Lung Biology in Health and Disease 121) 
10 Eddahibi S, Raffestin B, Clozel M, Levame M, and Adnot S. Protection from pulmonary hypertension with an orally active endothelin receptor antagonist in hypoxic rats. Am J Physiol Heart Circ Physiol 268: H828H835, 1995.

11 Feldkamp LA, Davis LC, and Kress JW. Practical cone-beam algorithm. J Opt Soc Am A 1: 612-619, 1984.

12 Finlay M, Barer GR, and Suggett AJ. Quantitative changes in the rat pulmonary vasculature in chronic hypoxia-relation to haemodynamic changes. Q J Exp Physiol 71: 151-163, 1986.

13 Fredberg JJ. Airway dynamics: recursiveness, randomness, and reciprocity in linear system simulation and parameter estimation. In: Respiratory Physiology: An Analytical Approach, edited by Chang MK and Paiva M. New York: Dekker, 1989, p. 167-194. (Lung Biology in Health and Disease 40)

14 Fredberg JJ. A modal perspective of lung response. J Acoust Soc Am 63: 962-966, 1978.

15 Frey U, Hislop A, and Silverman M. Branching properties of the pulmonary arterial tree during pre- and postnatal development. Respir Physiol Neurobiol 139: 179-189, 2004.

16 Herold CJ, Wetzel RC, Robotham JL, Herold SM, and Zerhouni EA. Acute effects of increased intravascular volume and hypoxia on the pulmonary circulation: assessment with high-resolution CT. Radiology 183: 655$662,1992$.

17 Hislop A and Reid L. New findings in pulmonary arteries of rats with hypoxia-induced pulmonary hypertension. Br J Exp Pathol 57: 542-554, 1976.

18 Horsfield K. Morphometry of the small pulmonary arteries in man. Circ Res 42: 593-597, 1978.

19 Horsfield K. Quantitative morphology and structure: functional correlations in the lung. Monogr Pathol 19: 151-159, 1978.

20 Jiang ZL, Kassab GS, and Fung YC. Diameter-defined Strahler system and connectivity matrix of the pulmonary arterial tree. J Appl Physiol 76: 882-892, 1994.

21 Karau KL, Johnson RH, Molthen RC, Dhyani AH, Haworth ST, Hanger CC, Roerig DL, and Dawson CA. Microfocal X-ray CT imaging and pulmonary arterial distensibility in excised rat lungs. Am J Physiol Heart Circ Physiol 281: H1447-H1457, 2001.

22 Karau KL, Molthen RC, Dhyani A, Haworth ST, Hanger CC, Roerig DL, Johnson RH, and Dawson 
CA. Pulmonary arterial morphometry from microfocal X-ray computed tomography. Am J Physiol Heart Circ Physiol 281: H2747-H2756, 2001.

23 Krenz GS and Dawson CA. Flow and pressure distributions in vascular networks consisting of distensible vessels. Am J Physiol Heart Circ Physiol 284: H2192-H2203, 2003.

24 Krenz GS and Dawson CA. Vessel distensibility and flow distribution in vascular trees. J Math Biol 44: 360374, 2002.

25 Lane KB, Machado RD, Pauciulo MW, Thomson JR, Phillips JA 3rd, Loyd JE, Nichols WC, and Trembath RC. Heterozygous germline mutations in BMPR2, encoding a TGF-beta receptor, cause familial primary pulmonary hypertension. The International PPH Consortium. Nat Genet 26: 81-84, 2000.

26 Linehan JH, Haworth ST, Nelin LD, Krenz GS, and Dawson CA. A simple distensible vessel model for interpreting pulmonary vascular pressure-flow curves. J Appl Physiol 73: 987-994, 1992.

$27 \mathrm{Liu} \mathrm{YH,} \mathrm{Hoffman} \mathrm{EA,} \mathrm{and} \mathrm{Ritman} \mathrm{EL.} \mathrm{Measurement} \mathrm{of} \mathrm{three-dimensional} \mathrm{anatomy} \mathrm{and} \mathrm{function} \mathrm{of} \mathrm{pulmonary}$ arteries with high-speed x-ray computed tomography. Invest Radiol 22: 28-36, 1987.

28 Machado RD, Pauciulo MW, Thomson JR, Lane KB, Morgan NV, Wheeler L, Phillips JA 3rd, Newman J, Williams D, Galie N, Manes A, McNeil K, Yacoub M, Mikhail G, Rogers P, Corris P, Humbert M, Donnai D, Martensson G, Tranebjaerg L, Loyd JE, Trembath RC, and Nichols WC. BMPR2 haploinsufficiency as the inherited molecular mechanism for primary pulmonary hypertension. Am J Hum Genet 68: 92-102, 2001.

29 Meyrick B, Hislop A, and Reid L. Pulmonary arteries of the normal rat: the thick walled oblique muscle segment. J Anat 125: 209-221, 1978.

30 Meyrick B and Reid L. Development of pulmonary arterial changes in rats fed Crotalaria spectabilis. Am J Pathol 94: 37-51, 1979.

31 Meyrick B and Reid L. Hypoxia-induced structural changes in the media and adventitia of the rat hilar pulmonary artery and their regression. Am J Pathol 100: 151-178, 1980.

32 Meyrick B and Reid L. Ultrastructural features of the distended pulmonary arteries of the normal rat. Anat Rec 193: 71-97, 1979.

33 Minnich B, Bartel H, and Lametschwandtner A. Quantitative microvascular corrosion casting by 2D- and 3Dmorphometry. Ital J Anat Embryol 106: 213-220, 2001. 
34 Motulsky HJ and Ransnas LA. Fitting curves to data using nonlinear regression: a practical and nonmathematical review. FASEB J 1: 365-374, 1987.

35 Ono S, Westcott JY, and Voelkel NF. PAF antagonists inhibit pulmonary vascular remodeling induced by hypobaric hypoxia in rats. J Appl Physiol 73: 1084-1092, 1992.

36 Partovian C, Adnot S, Eddahibi S, Teiger E, Levame M, Dreyfus P, Raffestin B, and Frelin C. Heart and lung VEGF mRNA expression in rats with monocrotaline- or hypoxia-induced pulmonary hypertension. Am J Physiol Heart Circ Physiol 275: H1948-H1956, 1998.

37 Rabinovitch M, Gamble W, Nadas AS, Miettinen OS, and Reid L. Rat pulmonary circulation after chronic hypoxia: hemodynamic and structural features. Am J Physiol Heart Circ Physiol 236: H818-H827, 1979.

38 Sasaki S, Kobayashi N, Dambara T, Kira S, and Sakai T. Structural organization of pulmonary arteries in the rat lung. Anat Embryol (Berl) 191: 477-489, 1995.

39 Singhal S, Henderson R, Horsfield K, Harding K, and Cumming G. Morphometry of the human pulmonary arterial tree. Circ Res 33: 190-197, 1973.

40 Voelkel NF and Tuder RM. Hypoxia-induced pulmonary vascular remodeling: a model for what human disease? J Clin Invest 106: 733-738, 2000.

41 Yen RT, Fung YC, and Bingham N. Elasticity of small pulmonary arteries in the cat. J Biomech Eng 102: 170$177,1980$.

42 Yen RT, Zhuang FY, Fung YC, Ho HH, Tremer H, and Sobin SS. Morphometry of cat's pulmonary arterial tree. J Biomech Eng 106: 131-136, 1984.

43 Young SL. An isolated perfused rat lung preparation. Environ Health Perspect 16: 61-66, 1976.

44 Zhuang FY, Fung YC, and Yen RT. Analysis of blood flow in cat's lung with detailed anatomical and elasticity data. J Appl Physiol 55: 1341-1348, 1983. 